\title{
Prediction Model of In-Band Blocking Interference under the Electromagnetic Radiation of Dual-Frequency Continuous Wave
}

\author{
Kai Zhao $\mathbb{D}^{1},{ }^{1}$ Guanghui Wei, ${ }^{1}$ Yaping Wang, ${ }^{2}$ and Xiaodong Pan $\mathbb{D}^{1}$ \\ ${ }^{1}$ National Key Laboratory on Electromagnetic Environment Effects, Shijiazhuang Campus of Army Engineering University, \\ Shijiazhuang 050003, China \\ ${ }^{2}$ Naval Aeronautical University, Yantai 264000, China \\ Correspondence should be addressed to Kai Zhao; oeczhao@126.com
}

Received 4 March 2020; Revised 20 May 2020; Accepted 16 June 2020; Published 15 July 2020

Academic Editor: Giuseppina Monti

Copyright (๑) 2020 Kai Zhao et al. This is an open access article distributed under the Creative Commons Attribution License, which permits unrestricted use, distribution, and reproduction in any medium, provided the original work is properly cited.

Two hypotheses for the blocking effect mechanisms under the electromagnetic radiation are presented, and the corresponding dualfrequency interference prediction models are proposed, which can be used to predict the working state of equipment under the condition that the single-frequency susceptibility is known. By taking a radar and a communication radio as the EUT, the experiments of dual-frequency continuous wave electromagnetic radiation are designed and carried out to verify the prediction models. The test results show that the sensitive bandwidth of blocking interference effect for the tested radar and the communication station is consistent with their working bandwidth, respectively. By substituting the test data into the proposed prediction models, the prediction errors of the models are less than $13 \%$. Moreover, it can be found that the tested radar is more sensitive to the peak value of electric field, and the tested communication station is more sensitive to the effective value of electric field.

\section{Introduction}

With the rapid development of information technology and wide application of electronic equipment, the electromagnetic environment is getting worse and worse, which is characterized by a variety of electromagnetic signals overlapped intensively in the space domain, time domain, frequency domain, and energy domain. The working reliability of electronic equipment is seriously threatened. Once the interference signal frequency falls in the operating bandwidth of the receiver, the interference will lead to the receiver saturation and loss of working ability, even causing damage to the receiver. Accurately predicting the survivability of electronic equipment in complex electromagnetic environment is the basic work to improve its electromagnetic protection performance, which has great application value.

At present, the electromagnetic radiation susceptibility test specified in many electromagnetic compatibility standards is still based on single-source radiation, which cannot fully reflect the possible impact of complex electromagnetic environment. It is likely that the frequency equipment will be interfered in the evaluation of safety range. Many scholars at home and abroad have pointed out the shortcomings of traditional radiation susceptibility test methods and carried out the corresponding research [1-3]. In the complex space electromagnetic environment, the tested equipment may be affected within the known single-frequency interference safety margin, and the existing single-frequency susceptibility test cannot meet the requirements $[4,5]$. There are limitations in the existing EMC test of vehicle functional safety, without considering the simultaneous interference, including dual-frequency or multifrequency continuous wave interference, RF superimposed electrostatic pulse, or conducted interference, and the multisource intermodulation interference is also ignored [6]. Some scholars proposed a test method of electromagnetic compatibility with multiple sources, which proves that the microwave darkroom and dual-source network analyzer are the best equipment to study the signal integrity problems caused by the leakage of intermodulation products [7]. The above literature points out the shortcomings of traditional research methods, but no specific solutions are proposed. Some scholars studied the 
interference prediction method of radio fuze under the electromagnetic radiation of dual-source continuous wave, but there are specific requirements for the frequency difference of the two interference signals [8]. An interference prediction method of communication station for dualsource continuous wave is proposed, but the method is only suitable for out-of-band interference [9]. Other related researches are mainly from the macro perspective, taking into account the factors that cause interference to the equipment in the complex electromagnetic environment and using the methods of expert evaluation and statistical analysis to evaluate the survivability of the equipment [10-17].

For this reason, based on the blocking jamming mechanism, two in-band dual-frequency prediction models of receiver are proposed, which are verified by the radar and communication station. Firstly, in Section 2, two assumptions about the mechanism of receiver blocking interference are proposed, and corresponding in-band dual-frequency prediction models are established. Then, in Sections 3 and 4, two experiment platforms are built to validate the prediction model with a certain type of radar and communication station as the subjects. Finally, some conclusions are drawn in Section 5.

\section{Two Types of Prediction Models}

Blocking interference is one of the effects of electromagnetic radiation field. It means that when strong interference signal and useful signal are joined to the RF front end simultaneously, the receiver gain decreases, even the useful signal cannot be received. Generally speaking, under the same interference frequency, the interference effect is positively correlated with the effective value of the interference signal field strength [18]. Some scholars pointed out that when the interference signal is out of band, if we want to cause effective interference to the receiver, the amplitude of its field strength needs to be large enough to make the nonlinear elements work in the special areas such as saturation and cutoff [9]. Therefore, two hypotheses are proposed for the blocking effect mechanism of the receiver: one is that the receiver is sensitive to the effective value of electromagnetic radiation field intensity, and the other is that the receiver is sensitive to the amplitude of electromagnetic radiation field intensity. Next, the above two assumptions will be used to establish the corresponding prediction model.

\subsection{Field Intensity Effective Value Sensitive Prediction Model.} It is assumed that the receiver is sensitive to the effective value of the electromagnetic radiation field intensity; that is, as long as the field intensity effective value of the interference signals is higher than the critical interference field intensity, it will cause effective interference to the receiver. The electromagnetic wave in space is generally coupled to the receiver through the antenna. Considering the single-frequency interference, the coupling voltage can be expressed as

$$
u_{r}(t)=u_{s}(t)+u_{j}(t)=A_{s} E_{s} \cos \omega_{s} t+A_{j} E_{j} \cos \omega_{j} t,
$$

where $E_{s}$ and $E_{j}$ are the field intensity amplitude of useful signal and interference signal in space; $\omega_{s}$ and $\omega_{j}$ are the angular frequency of useful signal and interference signal; and $A_{s}$ and $A_{j}$ are the amplitude-frequency coefficients, which can be considered as constants.

Considering that the interference is sensitive to the effective value of the field intensity; that is, it is sensitive to the effective value of the coupling voltage. The effective value of the voltage formed by the interference signal whose angular frequency is $\omega_{j}$ after frequency-selective filtering can be expressed as

$$
U_{e}=\frac{A_{j} E_{j}^{\prime}}{\sqrt{2}},
$$

where $E_{j}^{\prime}$ is the amplitude of the critical interference field intensity under the independent action of the interference signal with the angular frequency $\omega_{j}$. When $U_{e}$ reaches a certain threshold $U_{0}$, it will cause interference to the receiver; that is,

$$
\frac{A_{j} E_{j}^{\prime}}{\sqrt{2}}=U_{0} .
$$

When the receiver is subjected to in-band interference with angular frequencies $\omega_{1}$ and $\omega_{2}$ separately, the critical interference state satisfies the following formula:

$$
\left\{\begin{array}{l}
\frac{A_{1} E_{1}^{\prime}}{\sqrt{2}}=U_{0}, \\
\frac{A_{2} E_{2}^{\prime}}{\sqrt{2}}=U_{0},
\end{array}\right.
$$

where $A_{1}$ and $A_{2}$ are amplitude-frequency coefficients; $E_{1}^{\prime}$ and $E_{2}^{\prime}$ are the amplitudes of single-frequency critical interference field intensity. When the receiver is subject to the above dual-frequency interference at the same time, ignoring the contribution of interference intermodulation, the critical interference condition can be expressed as

$$
\left(\frac{A_{1} E_{1}}{\sqrt{2}}\right)^{2}+\left(\frac{A_{2} E_{2}}{\sqrt{2}}\right)^{2}=U_{0}^{2},
$$

where $E_{1}$ and $E_{2}$ are the field intensity amplitudes of the dual-frequency signals. From the combination of equations (4) and (5), there is

$$
\frac{E_{1}^{2}}{E_{1}^{\prime 2}}+\frac{E_{2}^{2}}{E_{2}^{\prime 2}}=1 .
$$

Therefore, the effect index of field intensity effective value sensitive prediction model can be defined as

$$
S_{1}=\frac{E_{1}^{2}}{E_{1}^{\prime 2}}+\frac{E_{2}^{2}}{E_{2}^{\prime 2}} \text {. }
$$

When $S_{1}<1$, it can be determined that the receiver works normally. When $S_{1} \geq 1$, it can be determined that the receiver is effectively interfered and cannot work normally. According to Parseval's theorem [19], equation (7) can also be written as 


$$
S_{1}=\frac{P_{1}}{P_{1}^{\prime}}+\frac{P_{2}}{P_{2}^{\prime}},
$$

where $P_{i}^{\prime}(i=1,2)$ is the average power of single-frequency critical interference of the receiver and $P_{i}(i=1,2)$ is the average power of in-band dual-frequency interference.

2.2. Field Intensity Amplitude Sensitive Prediction Model. Assuming that the receiver is sensitive to the amplitude of electromagnetic radiation field intensity, that is, as long as the field intensity amplitude of the interference signals is higher than the critical interference field intensity, it will cause effective interference to the receiver. The coupling voltage on the receiver due to the electromagnetic radiation in space is shown in equation (1). It can be seen from the above assumptions that the coupling voltage can replace the field strength to reflect the sensitive characteristics of the receiver. If the coupling voltage reaches a certain threshold $U_{0}$, the receiver will be interfered effectively. That is to say,

$$
A_{j} E_{j}^{\prime}=U_{0} \text {. }
$$

When the receiver is subjected to in-band interference with angular frequencies $\omega_{1}$ and $\omega_{2}$ separately, there is

$$
\left\{\begin{array}{l}
A_{1} E_{1}^{\prime}=U_{0}, \\
A_{2} E_{2}^{\prime}=U_{0} .
\end{array}\right.
$$

When the receiver is subject to the above dual-frequency interference at the same time, the maximum value of voltage amplitude can be expressed as $A_{1} E_{1}+A_{2} E_{2}$ under the harshest conditions. If the critical interference condition is satisfied, there is

$$
A_{1} E_{1}+A_{2} E_{2}=U_{0}
$$

From the combination of equations (10) and (11), there is

$$
\frac{E_{1}}{E_{1}^{\prime}}+\frac{E_{2}}{E_{2}^{\prime}}=1 .
$$

So, the effect index of field intensity amplitude sensitive prediction model can be defined as

$$
S_{2}=\frac{E_{1}}{E_{1}^{\prime}}+\frac{E_{2}}{E_{2}^{\prime}}
$$

Whether the receiver is effectively interfered can be determined according to the relationship between $S_{2}$ and 1 shown in Section 2.1. According to Parseval's theorem [19], the following equation still holds:

$$
S_{2}=\sqrt{\frac{P_{1}}{P_{1}^{\prime}}}+\sqrt{\frac{P_{2}}{P_{2}^{\prime}}} .
$$

\section{Continuous Wave Electromagnetic Radiation Experiment of a Certain Radar}

3.1. Experiment Layout. The radar under test is a Ku band stepped frequency continuous wave radar, with a working bandwidth of $\pm 100 \mathrm{MHz}$, which has the function of static target ranging. In the process of signal processing, the I/Q channel data collected by the radar is processed by inverse Fourier transform to obtain the one-dimensional range profile of the target echo, forming a peak spectrum at the target distance. The echo intensity of different targets is normalized. Typical detection image generated by the radar under test is shown in Figure 1.

The experiment is carried out in the open field, and the specific layout is shown in Figure 2. The signal generators 1 and 2 can, respectively, generate interference signals to feed the radiation antenna, and the spectrum analyzer monitors the interference power through the directional coupler. The gain of the radiation antenna has a higher flatness in the frequency band required by the subsequent experiment, and the change of gain here can be ignored. Adjust the position of the radiation antenna and the radar receiving antenna to meet the far-field conditions, and let the polarization direction of the radiation antenna be the same as that of the radar receiving antenna. $\mathrm{A} 50 \mathrm{~dB}$ attenuator is connected between the radar receiving antenna and the radar host, which makes the tested radar more vulnerable to interference and convenient for experiment. At the same time, the radiation antenna acts as the detection target of the radar.

Since the purpose of the experiment is to verify the proposed prediction model, the actual output power of the signal generator is used to replace the interference power received by the tested radar. It has been proved by other experiments that the above two are linear, so there is no additional error for the verification model. The same is true for the later experiments using communication stations.

\subsection{Susceptibility Criterion of Blocking Effect for the Radar} under Test. The susceptibility criterion is mainly used to verify the model, and it is required to accurately reflect whether the radar under test is disturbed. That is to say, in a certain area, the small change of the interference power will cause a large change of the susceptibility parameters of the equipment under test. According to the blocking effect mechanism, the radar will produce blocking effect under electromagnetic interference, thus reducing the dynamic range. For the radar under test, the minimum normalized level of range profile will increase due to the electromagnetic interference. That is to say, the dynamic range of the output range profile will be reduced. The dynamic range compression of range profile is determined by experiment, and the specific susceptibility criterion is given.

Build the test platform shown in Figure 2. Turn on the signal generator 1 to generate interference signal and increase the output power gradually. Detect and record the minimum value of interference power and normalized level after each radiation. Select different interference frequency offset (relative to the operating frequency), and repeat the test. The changes of the minimum value of normalized level with the interference field intensity under different frequency offsets are shown in Figure 3.

It can be seen from Figure 3 that the dynamic range of the radar is more sensitive to the interference power. Under the interference of different frequency offsets, the change 


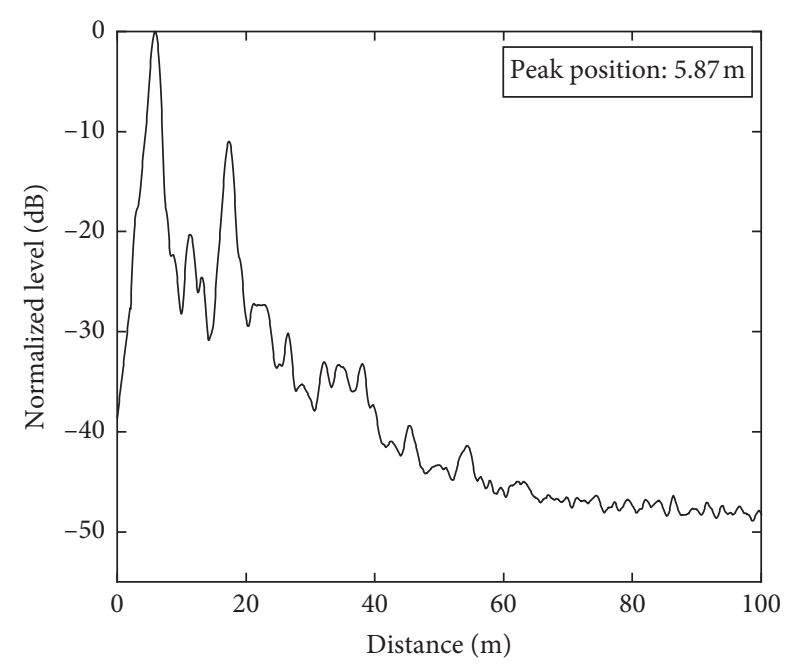

FIgURE 1: Image generated by the radar under test.

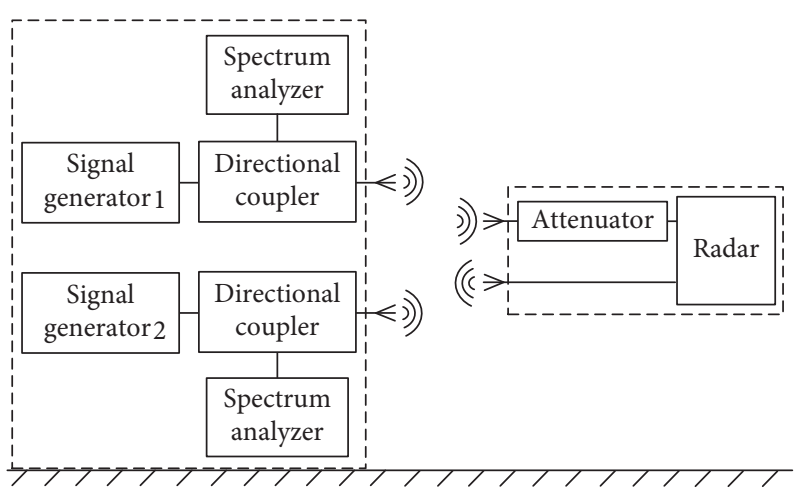

Figure 2: Layout of continuous wave electromagnetic radiation experiment on the radar.

rule of the dynamic range with the interference power is basically the same: the dynamic range compression increases gradually from zero with the increase of the interference power.

In addition, under the same interference power, the smaller the frequency offset, the more sensitive the radar under test, which conforms to the general cognition. It can be concluded from the radar equation that when the dynamic range is reduced by $12 \mathrm{~dB}$, the maximum detection range of the radar will be reduced by half, which can be used as a susceptibility criterion for the blocking effect of the radar.

The single-frequency susceptibility of the radar under test is shown in Figure 4. The sensitive bandwidth of electromagnetic radiation blocking effect of the radar is within $\pm 100 \mathrm{MHz}$, which is consistent with its working bandwidth. When the radiation frequency offset is about $\pm 100 \mathrm{MHz}$, the critical interference power increases sharply with a change of more than $30 \mathrm{~dB}$. When the radiation frequency offset is greater than $105 \mathrm{MHz}$, the critical interference power fluctuates and increases. It can be seen that using the dynamic range compression $12 \mathrm{~dB}$ as the susceptibility criterion can effectively reflect the interference state of the radar under test.

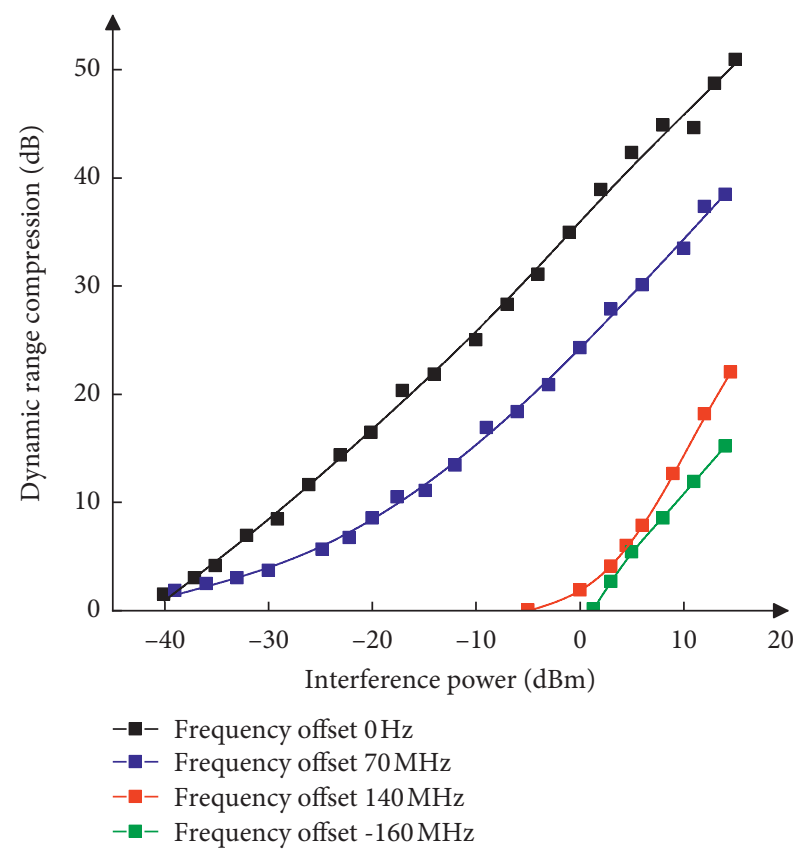

FIgURE 3: The dynamic range compression changes with the interference power under different frequency offsets.

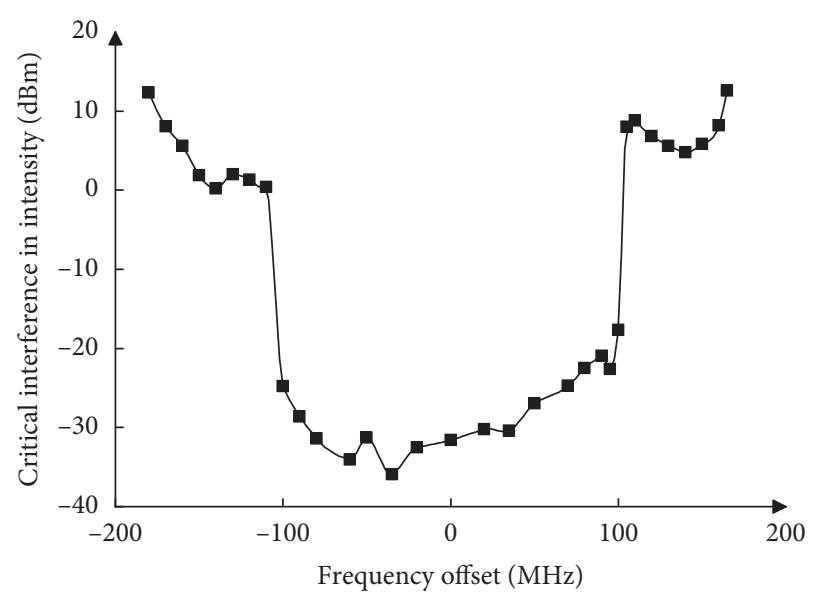

FIgURE 4: Single-frequency susceptibility of the radar under test.

3.3. Experimental Verification. In order to verify the correctness of the prediction model, the in-band dual-frequency electromagnetic radiation experiment was carried out. The experiment layout is shown in Figure 2, and the specific test steps are as follows:

(1) Set the signal generators 1 and 2 to output the interference signals with different frequency offsets. Test the critical interference power under the given single-frequency interference.

(2) Reduce the output power of the signal generator 1 and keep it unchanged. Gradually increase the power of the signal generator 2 until the radar under test reaches the critical jamming state, and record the corresponding power through the spectrum analyzer. 
TABLE 1: Critical interference power when the frequency offset of interference signal is $-20 \mathrm{MHz}$ and $40 \mathrm{MHz}$.

\begin{tabular}{lcccccc}
\hline & $\Delta f_{1}=-20 \mathrm{MHz}$ & $\Delta f_{2}=40 \mathrm{MHz}$ & $S_{1}$ & Error (\%) & $S_{2}$ & Error $(\%)$ \\
\hline Single-frequency critical interference power $(\mathrm{dBm})$ & -28.0 & -18.3 & - & - & - & - \\
\hline & -36.0 & -20.8 & 0.73 & 27 & 1.15 & 15 \\
Dual-frequency critical interference power $(\mathrm{dBm})$ & -34.0 & -21.9 & 0.69 & 31 & 1.16 & 16 \\
& -32.0 & -28.8 & 0.49 & 51 & 0.93 & 7 \\
\hline Average value & -30.0 & -30.9 & 0.69 & 31 & 1.03 & 3 \\
\hline
\end{tabular}

TABLE 2: Critical interference power when the frequency offset of interference signal is $-40 \mathrm{MHz}$ and $60 \mathrm{MHz}$.

\begin{tabular}{|c|c|c|c|c|c|c|}
\hline & $\Delta f_{1}=-40 \mathrm{MHz}$ & $\Delta f_{2}=60 \mathrm{MHz}$ & $S_{1}$ & Error $(\%)$ & $S_{2}$ & Error $(\%)$ \\
\hline Single-frequency critical interference power $(\mathrm{dBm})$ & -25.0 & -11.1 & - & - & - & - \\
\hline \multirow{4}{*}{ Dual-frequency critical interference power $(\mathrm{dBm})$} & -33.0 & -14.2 & 0.65 & 35 & 1.10 & 10 \\
\hline & -31.0 & -16.0 & 0.57 & 43 & 1.08 & 8 \\
\hline & -29.0 & -18.3 & 0.59 & 41 & 1.07 & 7 \\
\hline & -27.0 & -23.9 & 0.68 & 32 & 1.03 & 3 \\
\hline Average value & - & - & 0.62 & 38 & 1.07 & 7 \\
\hline
\end{tabular}

(3) Increase the power of signal generator 1 in a certain step and keep it unchanged. Adjust the output power of signal generator 2 until the radar reaches the critical interference state, and record the corresponding power.

Two groups of dual-frequency interference combinations are selected, and their frequency offsets are $-20 \mathrm{MHz}$ and $40 \mathrm{MHz} ;-40 \mathrm{MHz}$ and $60 \mathrm{MHz}$, respectively. The test results are substituted into (8) and (14) to obtain the effect indices $S_{1}$ and $S_{2}$, as shown in Tables 1 and 2 .

It can be seen from Tables 1 and 2 that the radar under test is sensitive to the dual-frequency signals. The critical interference power under the dual-frequency jamming is lower than the critical power of each single-frequency interference. When the interference frequency offset is -20 and $40 \mathrm{MHz}$, the effect index $S_{2}$ is basically close to 1 , with the maximum error of $16 \%$ and the average error of $7 \%$. The effect index $S_{1}$ is basically between 0.5 and 0.7 , with the maximum error of $43 \%$ which is higher than $S_{2}$. When the interference frequency offset is -40 and $60 \mathrm{MHz}$, the calculated $S_{2}$ is also close to 1 . Thus, the field intensity amplitude sensitive model is suitable for the radar under test, and the test verifies the correctness of the model.

\section{Continuous Wave Electromagnetic Radiation Experiment of a Certain Communication Station}

4.1. Experiment Layout. The specific experiment layout is shown in Figure 5. In the experiment, the sine wave signal generated by the signal generator is used as the interference source. The polarization direction of the interference source is the same as that of the receiving antenna of communication station. When the dual-frequency interference experiment is carried out, two signal generators with the same model are used to feed the radiation antenna through the power synthesizer. By adjusting the power of the signal generator,

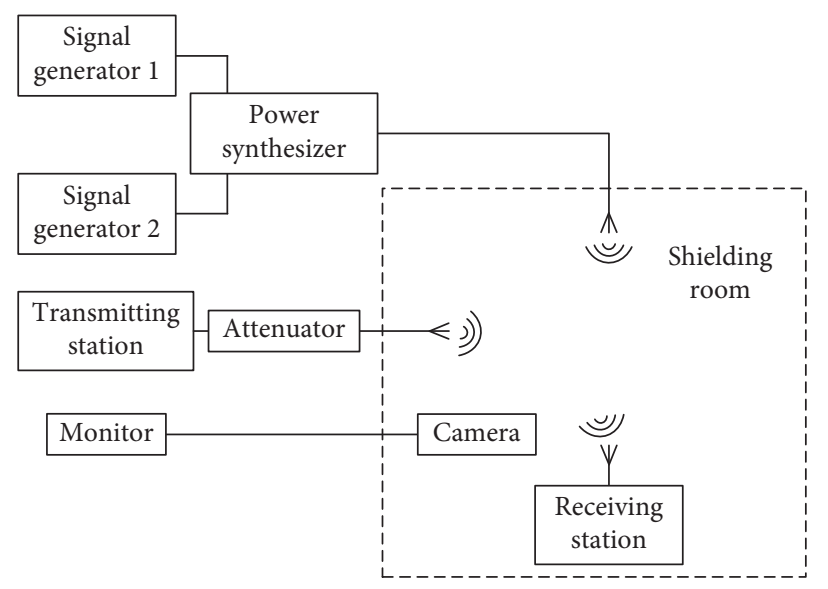

FIGURE 5: Layout of continuous wave electromagnetic radiation experiment on the communication station.

observe the working state of the radio station under test. Note that the radio station has three kinds of working modes: highpower, medium-power, and low-power. Different transmitting power is mainly adapted to different working distances. During the experiment, the radio station is selected to work in the low-power mode. In order to simulate the long-distance communication conditions, an attenuator is connected at the output terminal of the transmitting station.

\subsection{Susceptibility Criterion of Blocking Effect for the} Communication Station under Test. In general, the bit error rate can be used to judge the interference degree of the communication station. The bit error rate is directly related to the strength of interference signal. In order to determine the susceptibility criterion, the bit error rate at the maximum change rate in the field strength and bit error rate curve is selected as the critical interference criterion. The result shows that when the bit error rate reaches $10 \%$, the radio station will be in a critical interference state [20]. Set up the experimental platform according to Figure 5, and test the 


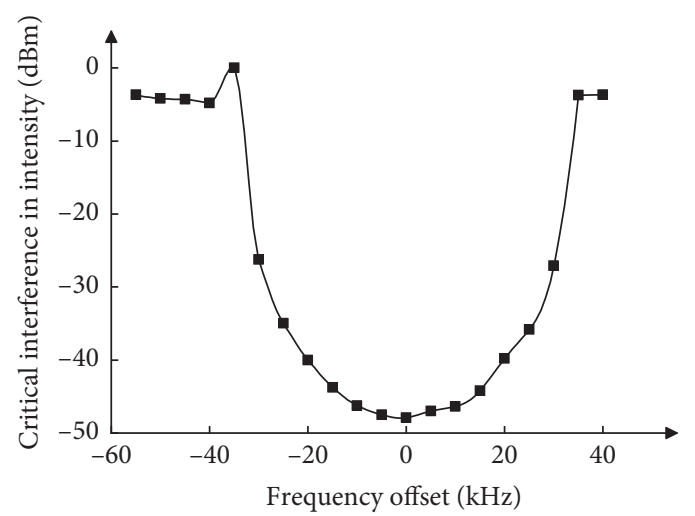

(a)

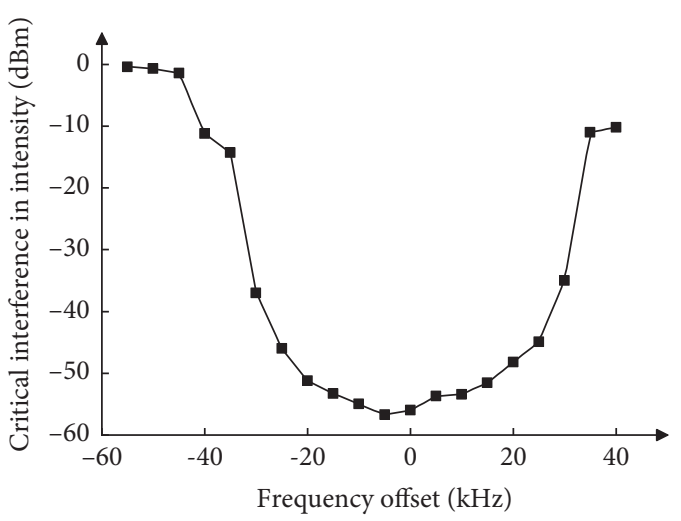

(b)

FIGURE 6: Single-frequency susceptibility of the communication station under test. (a) The communication station is working at $80 \mathrm{MHz}$. (b) The communication station is working at $40 \mathrm{MHz}$.

TABLE 3: Critical interference power when the communication station is working at $80 \mathrm{MHz}$.

\begin{tabular}{|c|c|c|c|c|c|c|}
\hline & $\Delta f_{1}=-20 \mathrm{kHz}$ & $\Delta f_{2}=10 \mathrm{kHz}$ & $S_{1}$ & Error (\%) & $S_{2}$ & Error $(\%)$ \\
\hline Single-frequency critical interference power $(\mathrm{dBm})$ & -1.2 & -2.6 & - & - & - & - \\
\hline \multirow{6}{*}{ Dual-frequency critical interference power $(\mathrm{dBm})$} & -12.0 & -2.6 & 1.06 & 6 & 1.28 & 28 \\
\hline & -10.0 & -2.7 & 1.09 & 9 & 1.34 & 34 \\
\hline & -8.0 & -2.8 & 1.14 & 14 & 1.42 & 42 \\
\hline & -6.0 & -3.2 & 1.18 & 18 & 1.50 & 50 \\
\hline & -4.0 & -3.9 & 1.25 & 25 & 1.58 & 58 \\
\hline & -2.0 & -7.3 & 1.17 & 17 & 1.49 & 49 \\
\hline Average value & - & - & 1.13 & 13 & 1.41 & 41 \\
\hline
\end{tabular}

TABLE 4: Critical interference power when the communication station is working at $40 \mathrm{MHz}$.

\begin{tabular}{|c|c|c|c|c|c|c|}
\hline & $\Delta f_{1}=-25 \mathrm{kHz}$ & $\Delta f_{2}=5 \mathrm{kHz}$ & $S_{1}$ & Error (\%) & $S_{2}$ & Error (\%) \\
\hline Single-frequency critical interference power $(\mathrm{dBm})$ & -1.5 & -8.2 & - & - & - & - \\
\hline \multirow{5}{*}{ Dual-frequency critical interference power $(\mathrm{dBm})$} & -12.0 & -8.3 & 1.07 & 7 & 1.29 & 29 \\
\hline & -10.0 & -9.1 & 0.95 & -5 & 1.28 & 28 \\
\hline & -8.0 & -9.7 & 0.93 & -7 & 1.31 & 31 \\
\hline & -6.0 & -8.5 & 1.29 & 29 & 1.56 & 56 \\
\hline & -4.0 & -14.4 & 0.81 & -19 & 1.25 & 25 \\
\hline Average value & - & - & 1.01 & 13 & 1.34 & 34 \\
\hline
\end{tabular}

single-frequency susceptibility of the communication radio station under test. Select two working frequency points of the communication radio: $80 \mathrm{MHz}$ and $40 \mathrm{MHz}$. The specific test method is similar to that given in Section 2.2 and will not be described in detail. The test results are shown in Figure 6.

According to Figure 6, the sensitive bandwidth of electromagnetic radiation blocking effect of the communication under test is about $60 \mathrm{kHz}$, which is consistent with its working bandwidth. The difference of the critical interference power value between in-band and out-of-band is at least $40 \mathrm{~dB}$, which indicates that the bit error rate in the link test can be used as the criterion to reflect whether the radio station is interfered.

4.3. Experimental Verification. In order to verify the correctness of the prediction model, the in-band dual-frequency electromagnetic radiation experiment is carried out on the communication station.
Adjust the working frequency of the communication station to $80 \mathrm{MHz}$, and the two interference signals are generated by signal generators 1 and 2, respectively. The frequency offsets are $-20 \mathrm{kHz}$ and $10 \mathrm{kHz}$. The test method is similar to that in Section 3.3 and will not be repeated. The results are shown in Table 3.

The interference threshold in Table 3 is inconsistent with that in Figure 6, because the attenuators connected between the transmitting station and the antenna are different in the two tests. It can be seen from Table 3 that the communication station under test is also sensitive to the dual-frequency interference. When the interference frequency offset is $-20 \mathrm{kHz}$ and $10 \mathrm{kHz}$, the effect index $S_{1}$ is basically close to 1 , and its maximum error is $25 \%$, with an average of $13 \%$. However, the maximum error of effect index $S_{2}$ is $58 \%$, with an average of $41 \%$. The result shows that the communication station under test is more sensitive to the effective value of field intensity. 
In order to further verify the prediction model, another working frequency of the communication station under test is set at $40 \mathrm{MHz}$, and the frequency offsets of the two interference signals are $-25 \mathrm{kHz}$ and $5 \mathrm{kHz}$, respectively. According to the above method, the experiment results are shown in Table 4 .

It can be seen from Table 4 that the communication station is also sensitive to the dual-frequency interference when the working frequency is $40 \mathrm{MHz}$, and its effect index $S_{1}$ is also close to the theoretical value 1 . According to the above data, the field intensity effective value sensitive prediction model is suitable for the radio station under test.

\section{Conclusion}

In order to accurately predict the survivability of the electronic equipment under the in-band dual-frequency electromagnetic environment, two hypotheses on the mechanism of the electromagnetic radiation blocking effect to the receiver are presented, and corresponding prediction models are established, respectively. Taking a radar and a communication station as the tested object, the prediction models are verified. The conclusions are as follows:

(1) The susceptibility criterion of blocking effect for the equipment under test is given. $12 \mathrm{~dB}$ drop in the dynamic range and $10 \%$ bit error rate are used as the blocking effect susceptibility criterion of radar and communication station, respectively. The sensitive bandwidth of them is basically consistent with their respective working bandwidth.

(2) The radar under test is more sensitive to the amplitude of the interference field intensity. The field intensity amplitude sensitive prediction model can be used in the prediction of dual-frequency blocking effect on it.

(3) The communication station under test is more sensitive to the effective value of the interference field intensity. The field intensity amplitude sensitive prediction model can be used in the prediction of dual-frequency blocking effect on it.

(4) The prediction models proposed in this paper can be further extended to multifrequency interference in theory. However, due to the limitations of test conditions, no further verification is carried out, which needs to be further explored in the next study.

\section{Data Availability}

The data used to support the findings of this study are available from the corresponding author upon request.

\section{Conflicts of Interest}

The authors declare that they have no conflicts of interest regarding the publication of this paper.

\section{Acknowledgments}

This work was supported by the Natural Science Foundation of Hebei Province (E2019506032).

\section{References}

[1] "GJB 151B-2013 Electromagnetic emission and susceptibility requirements and measurements for military and subsystems," 2013.

[2] International Electrotechnical Commission, IEC/TS 61000-12 Electromagnetic Compatibility (EMC)-Methodology for the Achievement of the Functional Safety of Electrical and Electronic with Regard to Electromagnetic Phenomena, International Electrotechnical Commission, Geneva, Switzerland, 2008.

[3] United States Department of Defense, "MIL-STD-461F requirements for the control of electromagnetic interference characteristics of subsystems and equipment," 2007.

[4] A. Duffy, K. Armstrong, and A. Orlandi, "Preliminary study of a reverberation chamber method for multiple-source testing using intermodulation," IET Science, Measurement \& Technology, vol. 4, no. 1, pp. 21-27, 2010.

[5] W. Grommes and K. Armstrong, "Developing immunity testing to cover intermodulation," in Proceedings of the 2011 IEEE International Symposium on Electromagnetic Compatibility (EMC), pp. 999-1004, Long Beach, CA, USA, August 2011.

[6] E. K. Armstrong, "EMC for the functional safety of automobiles why EMC testing is insufficient, and what is necessary," in Proceedings of the IEEE International Symposium on EMC, pp. 1-6, Detroit, MI, USA, August 2008.

[7] A. Biondi, L. Vallozzi, H. Rogier, D. D. Zutter, and F. Declercq, "Electromagnetic compatibility aware design and testing of intermodulation distortion under multiple co-located sources illumination," IET Science, Measurement \& Technology, vol. 6, no. 2, pp. 105-112, 2012.

[8] G. Zhao, G. Wei, X. Lu et al., "Prediction method of irradiation effects of dual sine waves on certain radio fuze," Journal of Academy of Armored Force Engineering, vol. 28, no. 5, pp. 53-56, 2014.

[9] Y. Wang, G. Wei, X. Pan et al., "Out-of-band dual-frequency jamming prediction model and experiment for communication stations," Acta Electronica Sinica, vol. 47, no. 4, pp. 826-831, 2019.

[10] C. Wang, Y. Sun, and C. Wu, "Complex electromagnetic environment surface to air missile system viability evaluation," Ship Electronic Engineering, vol. 17, no. 8, pp. 68-72, 2009.

[11] B. Li, J. Dong, C. Lv, and Y. Li, "Evaluating the complexity degree of electromagnetic environment utilizing morphological covering based fractal dimension," in Proceedings of the IEEE International Conference on Electronic Measurement \& Instruments, pp. 617-620, Harbin, China, August 2014.

[12] S. J. Zhang, D. L. Su, J. Wang, and R. Wang, "Complexity evaluation method of electromagnetic environment oriented to communication equipment based on statistic theory," Applied Mechanics and Materials, vol. 568-570, pp. 13311335, 2014.

[13] C. Zhou, Z. Wang, T. Liu, S. Zhao, and Z. Liang, "Low dropout linear regulator electromagnetic interference damage model based on BP neural network," High Voltage Engineering, vol. 42, no. 3, pp. 973-979, 2016.

[14] D. Zhang, Y. Chen, E. Cheng, and B. Du, "Research on dynamic electromagnetic susceptibility for electromagnetic interference prediction of UAV information link," High Voltage Engineering, vol. 45, no. 2, pp. 665-672, 2019.

[15] B. Yin, S. Wang, Y. He et al., "Electromagnetic environment complex evaluation algorithm based on fast s-transform and 
time-frequency space model," Journal of Electronics and Information Technology, vol. 41, no. 1, pp. 195-201, 2019.

[16] Y. Xu, Research on the Safety of Electro-Explosive Products with Complex Electromagnetic Environment, Harbin Institute of Technology, Harbin, China, 2019.

[17] G. Wei, L. Geng, and X. Pan, "Mechanism of electromagnetic radiation effects for communication equipment," High Voltage Engineering, vol. 40, no. 9, pp. 2685-2692, 2014.

[18] W. Li, G.-H. Wei, X.-D. Pan, X.-F. Lu, H.-J. Wan, and H. Li, "Electromagnetic radiation effects forecasting method about in-band dual-frequency continuous wave for typical communication equipment," Systems Engineering and Electronics, vol. 38, no. 11, pp. 2474-2480, 2016.

[19] L. M. Surhone, M. T. Tennoe, S. F. Henssonow et al., "Parseval's theorem," Betascript Publishing, vol. 21, no. 1, pp. 134-148, 2010.

[20] W. Li, G. Wei, X. Pan et al., "Research on electromagnetic susceptibility criterion for typical communication equipment," Journal of Microwaves, vol. 32, no. 6, pp. 70-75, 2016. 